\title{
USO DE EXTRATOS COMERCIAIS DE CARVALHO PARA PRODUÇÃO DE AGUARDENTE
}

\author{
Usage of Commercial Oak Extracts to Produce \\ Alcoholic Beverage
}

\author{
Agenor Maccari Jr. \\ Professor do Departamento de Solos e Engenharia Agrícola, Universidade Federal do Paraná, \\ Curitiba - PR. E-mail: maccari@ufpr.br. \\ Fabiula M. Stella \\ Agrônoma, Cambona Tecnologia Agroindustrial, Curitiba - PR. E-mail: fmstella@cambona.com.br. \\ Lauren D. B. Roncato-Maccari \\ Agrônoma, Cambona Tecnologia Agroindustrial, Curitiba - PR. E-mail: 1roncato@cambona.com.br.
}

\begin{abstract}
Resumo
Compostos de madeiras têm sido utilizados na elaboração de bebidas alcoólicas como alternativa ao envelhecimento tradicionalmente realizado em tonéis de carvalho. Neste trabalho, quatro diferentes extratos de carvalho, comercializados para produção de bebidas alcoólicas, foram adicionados à cachaça branca, visando agregar propriedades sensoriais como coloração, sabor e aroma. As cachaças bonificadas com os quatro extratos foram analisadas quanto à acidez, ganho e estabilidade da coloração. Análises sensoriais também foram executadas. Os resultados mostraram que a adição dos extratos utilizados resultou em aumento de acidez da aguardente, bem como promoveu incrementos nos atributos sensoriais, considerando a cachaça branca usada, com diferenças na estabilidade do produto final.
\end{abstract}

Palavras-chave: Cachaça; Extrato de carvalho; Aguardente composta.

\begin{abstract}
Wood compounds have been used in the elaboration of alcoholic beverages, alternatively to beverage ageing on oak barrels. In the present work, four commercial oak extracts were added to the "cachaça", the brazilian sugar cane spirit, in order to aggregate sensorial properties such as color, taste and flavor. The new composed beverages were submitted to sensorial analysis. In addition, acidity, coloration increase and persistence were analyzed. The results showed that the oak extracts increased the acidity of the composed beverage, as well as enhanced the sensorial properties of the original spirit. Differences between extracts were observed, mainly in stability of the final alcoholic beverage.
\end{abstract}

Keywords: Sugar cane spirit; Oak extract; Alcoholic beverage. 


\section{INTRODUÇÃO}

O Brasil, como outras localidades do mundo, possui uma bebida genuína, a cachaça, uma aguardente obtida do caldo cana-de-açúcar (Saccharum officinarum), bebida tipicamente brasileira, que recebe várias denominações regionais, como caninha, pinga e branquinha (VALSECHI, 1962; NOVAIS, 1974). Apesar das várias denominações, a partir de 2001, "cachaça" tornou-se a denominação oficial e exclusiva da aguardente de cana produzida no Brasil. As características da cachaça, segundo o Decreto n. ${ }^{\circ}$ 4.062/01, são: bebida obtida pela destilação do mosto fermentado do caldo de cana-de-açúcar; graduação alcoólica de 38 a $48 \%$ vol a $20^{\circ} \mathrm{C}$; propriedades sensoriais peculiares; pode ser adicionada de açúcares até $6 \mathrm{~g} / \mathrm{L}$, expressos em sacarose.

Segundo Maia (2005), o Brasil constitui-se em um dos maiores mercados de bebidas destiladas, com uma produção anual estimada em 1,5 bilhão de litros e o consumo per capita de 11 litros/habitante/ano.

O envelhecimento é fundamental para que a bebida adquira as características sensoriais desejadas pelos consumidores, pois por melhor que sejam realizadas as etapas de produção (obtenção e processamento da matéria-prima, fermentação e destilação), o destilado obtido apresentará sabor seco, ardente e um aroma não muito agradável (BOZA; OETTERER, 1999, REAZIN, 1981). As características sensoriais desejáveis na bebida, segundo Almeida et al. (1947), são resultantes de reações que ocorrem naturalmente no produto, mas também por influência do material do tonel de envelhecimento, o qual transfere compostos para a aguardente, promovendo mudanças químicas, físicas e sensoriais no produto ali armazenado. Nishimura e Matsuyama (1989) relatam que óleos voláteis, substâncias tânicas, açúcares e glicerol, ácidos orgânicos não voláteis e esteróides são os principais compostos que modificam o aroma, o sabor e a coloração da bebida.

Estudos comprovam que a bebida envelhecida tem maior aceitação. Cardello e Faria (1998), em estudo para traçar o perfil sensorial da aguardente de cana durante o envelhecimento em tonéis de carvalho, detectaram que as aguardentes com maior tempo de envelhecimento foram mais bem avaliadas, provavelmente devido à maior contribuição para aroma, sabor e cor da bebida.

Entretanto, além de vantagens, o processo de envelhecimento apresenta alguns problemas como: a evaporação da cachaça; a concentração de compostos indesejáveis; e a dificuldade na padronização do produto final, decorrente de transformações que ocorrem nos tonéis pelo tempo de uso. Além destes problemas, deve-se considerar ainda o alto custo da madeira e o tempo que a bebida deve ficar armazenada.

Nesse contexto, diversas pesquisas têm sido desenvolvidas com o objetivo de acelerar, melhorar ou substituir o processo tradicional de envelhecimento de cachaça em barris de madeira. O estudo do armazenamento da cachaça em recipientes de vidro contendo raspas de madeira, isoladamente ou em conjunto, com aeração periódica, foi realizado por Queiroz (1998). Coutrim, Chaves e Brandão (2003) testaram a microxigenação da bebida em processo semelhante ao já utilizado no envelhecimento de vinhos. Já Forlin (2005) pesquisou a armazenagem de cachaças em recipientes de polietilenotereftalato (PET) com a adição de extrato de carvalho.

O carvalho europeu (Quercus alba) é a madeira mais utilizada para envelhecer bebidas alcoólicas, pelas características conferidas ao produto. Apresenta grande uso na Europa, mas segundo Dias et al. (1998), Duarte et al. (2000) e Yokota et al. (2003), há uma grande dificuldade para obtenção de carvalho europeu no Brasil.

A extração de compostos de madeiras, bem como de ervas, raízes, tubérculos, folhas e flores, é uma prática adotada na elaboração de muitas bebidas alcoólicas, visando associar propriedades sensoriais específicas de cor, aroma, sabor e agregar propriedades funcionais estimulantes e digestivas (TRITTON, 1975). A obtenção de extratos hidroalcoólicos de madeira para posterior utilização na maturação de bebidas destiladas tem mostrado viabilidade para a redução dos períodos tradicionais de envelhecimento em tonéis. O uso de extratos ou bonificadores, elaborados a partir da própria madeira, apresenta vantagens: 1) proporciona melhor controle e direcionamento de pré-tratamentos à madeira, 2) aumenta a eficiência relativa de extração, 3) possibilita a incorporação na bebida de compostos desejados da madeira, 4) permite melhor controle no desenvolvimento do perfil sensorial da bebida, 5) racionaliza o uso de madeira, 6) reduz as perdas da bebida por volatilização e os custos relativos de instalações físicas (ZIMLICH; JOSEPH, 2000; MARTINEZ et al., 1997; BOWEN et al., 1994). 
O objetivo deste trabalho foi avaliar os efeitos da adição de quatro diferentes extratos comerciais de carvalho à cachaça. A estabilidade dos produtos bonificados (aguardentes compostas) foi avaliada em relação ao ganho e persistência da coloração, acidez da bebida e atributos sensoriais.

\section{MATERIAL E MÉTODOS}

Neste trabalho, foram avaliados quatro diferentes extratos de carvalho, sendo três deles líquidos e um em pó. Esses extratos foram fornecidos pela empresa AEB Latino Americana S/A. Na Tabela 1 encontram-se os nomes comerciais dos produtos utilizados, suas especificações, bem como as doses utilizadas.

TABELA 1 - Extratos de carvalho, composição, doses

Table 1 - Oak extracts, composition, dosage

\begin{tabular}{lllc}
\hline Extrato & Composição & Dose & Código \\
\hline Ellagitan ${ }^{\circledR}$ Chene ${ }^{\circledR}$ & $\begin{array}{l}\text { Tanino elágico de origem } \\
\text { lenhosa de carvalho }\end{array}$ & $0,25 \mathrm{~g} / \mathrm{L}$ & CA1 \\
& $\begin{array}{l}\text { Tanino elágico de origem } \\
\text { Ellagitan }{ }^{\circledR} \text { Liquid }\end{array}$ & $0,50 \mathrm{~g} / \mathrm{L}$ & CA2 \\
& $\begin{array}{l}\text { Tanino enológico em solução } \\
\text { Ellagitan }{ }^{\circledR} \text { Barrique }\end{array}$ & $0,25 \mathrm{~g} / \mathrm{L}$ & LA1 \\
& $\begin{array}{l}\text { aquosa } \\
\text { Tllagitan }{ }^{\circledR} \text { Pó }\end{array}$ & $0,25 \mathrm{~g} / \mathrm{L}$ & LA2 \\
& vegetal & $0,50 \mathrm{~g} / \mathrm{L}$ & BA2 \\
& $0,25 \mathrm{~g} / \mathrm{L}$ & PA1 \\
\hline
\end{tabular}

Os extratos foram adicionados à cachaça branca, padrão comercial, com graduação alcoólica de $44,7^{\circ} \mathrm{GL}$, em duas doses $(0,25 \mathrm{~g} / \mathrm{L}$ e $0,5 \mathrm{~g} / \mathrm{L})$. O produto gerado, a aguardente composta, foi armazenado durante 90 dias em garrafas de vidro, mantidas à temperatura ambiente $\left(20-25^{\circ} \mathrm{C}\right)$.

A avaliação da coloração foi realizada diariamente até o décimo quinto dia, quando passaram a ser realizadas em intervalos de sete dias. As análises foram realizadas utilizando espectrofotômetro FEMTO 700 Plus e a leitura de absorbância feita a $430 \mathrm{~nm}$.

A análise da acidez foi realizada ao final do experimento, pois o período de 90 dias é insuficiente para mudanças significativas nesse parâmetro. A determinação da acidez foi realizada por titulação com solução de hidróxido de sódio 0,05N até coloração rósea clara (MAIA, 2005).

Após 90 dias, os produtos obtidos com a adição dos extratos foram submetidos à análise sensorial. Essa análise foi realizada em um painel composto por quatro julgadores treinados, sendo feita análise descritiva dos atributos de aparência, sabor e aroma (FERREIRA, 1999). A análise mostrou que a menor dose utilizada nos ensaios não contribuiu de forma perceptível com relação ao aroma e ao sabor da bebida, impossibilitando a caracterização e identificação dos atributos. Assim, o ensaio sensorial foi realizado com os tratamentos que receberam a dose maior $(0,5 \mathrm{~g} / \mathrm{L})$. 


\section{RESULTADOS E DISCUSSÃO}

\section{Coloração desenvolvida após adição dos extratos de carvalho}

A cor é um parâmetro determinante na aceitabilidade da aguardente de cana, (CARDELLO; FARIA, 1997). A figura 1 mostra as curvas de ganho de cor da aguardente para cada um dos extratos de carvalho, para as duas doses utilizadas. Os resultados mostram que houve um grande ganho de cor até o décimo quinto dia, para todos os extratos, nas duas doses utilizadas. Após esse período, o ganho de coloração foi mais lento, sendo possível identificar uma tendência de estabilização na coloração. Apesar de serem extratos diferentes, é possível afirmar que o comportamento de incremento da coloração da bebida ao longo do tempo é semelhante.

Os resultados mostram que todos os quatro extratos contribuíram em incremento de cor para a cachaça branca. Entretanto, esse aumento se dá de maneira diferenciada para cada extrato. Os tratamentos com Ellagitan Pó (PA) geraram os maiores ganhos de cor (em torno de 6 vezes a mais), nas duas doses testadas $(0,25$ e $0,5 \mathrm{~g} / \mathrm{L})$. Apesar do uso dos demais extratos também levarem a aumentos de coloração, esse se dá em níveis bem menores.

Além da variação na intensidade da cor, houve variação na cinética deste processo. Assim, as amostras de cachaça branca que receberam o Ellagitan Barrique e o Ellagitan Chene apresentaram um grande acréscimo de cor logo após a adição do extrato. Porém, a partir do 5o dia, o incremento de cor se tornou menos pronunciado. Cabe destacar que, com o tempo, esse processo passou a ser mais lento nos tratamentos com Ellagitan Barrique. Para os dois outros extratos, Ellagitan Liquid e Ellagitan Pó, houve um grande incremento de coloração nos primeiros 15 dias avaliados, sendo que o Ellagitan Liquid mostrou um ganho não tão acentuado (cerca de 4 vezes) quando comparado com o Ellagitan Pó. Observou-se que todas as variações mencionadas ocorreram para as duas doses utilizadas de extrato.

Um dos principais problemas decorrentes da adição de compostos químicos à cachaça diz respeito à estabilidade da bebida e a formação de precipitados. A utilização de extratos em bebidas destiladas altera a atividade relativa e a concentração de frações de solutos hidrofóbicos de baixo peso molecular (solúveis em etanol), influindo diretamente na estabilidade da bebida.

A formação de precipitados foi mais intensa no tratamento com o extrato Ellagitan Chene do que nos demais tratamentos. Após 5 dias da adição desse extrato à cachaça, foi observada a formação de precipitado nas garrafas para as duas doses avaliadas conforme figura 1. Apesar de menos intensa, decorridos 10 dias do início dos ensaios, foi observada formação de precipitados também no tratamento com Ellagitan Barrique na dose de $0,5 \mathrm{~g} / \mathrm{L}$. Nos outros dois tratamentos (Ellagitan Liquid e Ellagitan Pó), não houve formação de precipitado.

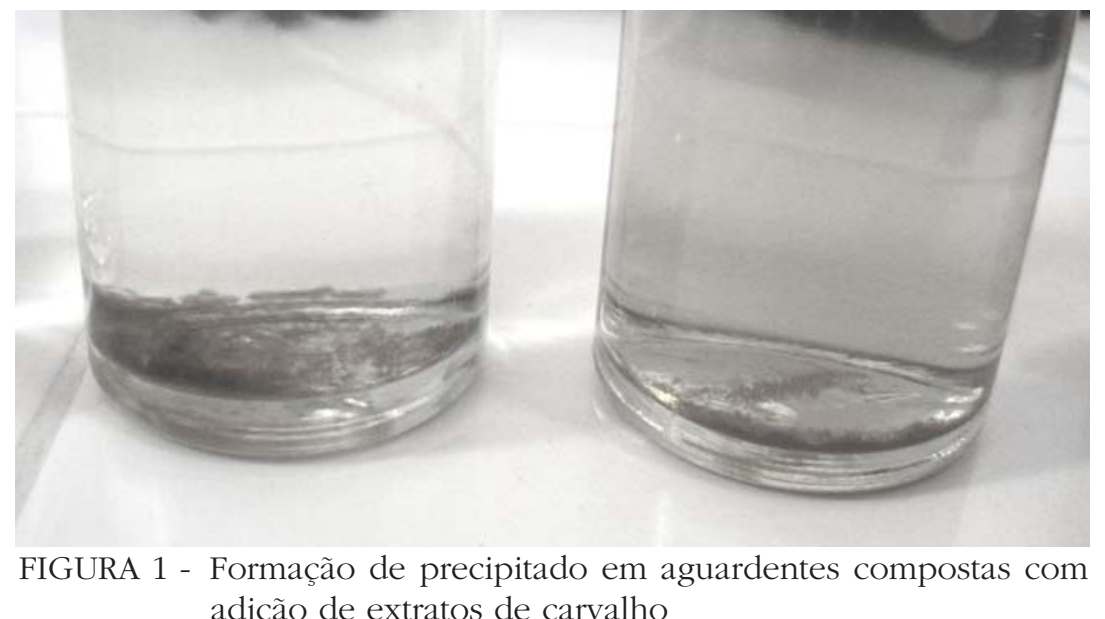
adição de extratos de carvalho

Figure 1 - Illustration of the precipitate formation in sugar cane spirit after oak extract addition 


\section{Acidez da cachaça adicionada dos extratos de carvalho}

A acidez é um parâmetro de qualidade no processo, indicando a ocorrência de contaminantes (MACCARI JR., 2005). Nesse sentido, a legislação vigente estabelece o limite máximo como sendo de 150 mg de ácido acético/100 mL de cachaça (BRASIL, 1974). Porém, a acidez pode ser um fator desejável, se dentro de certos limites, contribuindo para o sabor da aguardente, bem como participando da formação de aromas (esterificação) durante o envelhecimento (MAIA, 2005).

A tabela 2 mostra os resultados referentes à acidez da aguardente após 90 dias da adição dos extratos. A cachaça branca utilizada apresenta acidez baixa (37,7 mg ácido acético/100 mL de cachaça) se considerados os valores usuais (MAIA, 2005). Os resultados mostram que a adição de extrato de carvalho aumenta a acidez da aguardente elaborada.

TABELA 2 - Resultados da determinação de acidez nas amostras de aguardente, 90 dias após adição dos extratos de carvalho

Table 2 - Sugarcane spirit acidity results, 90 days after oak extracts addition

\begin{tabular}{lcc}
\hline Extrato & Dose utilizada & Acidez \\
& (mg ácido acético/100 $\mathrm{ml}$ cachaça)
\end{tabular}

$\begin{array}{llc}\text { Testemunha Cachaça branca } & 0,00 \mathrm{~g} / \mathrm{L} & 37,7 \\ \text { Ellagitan Chene } & 0,25 \mathrm{~g} / \mathrm{L} & 40,8 \\ & 0,50 \mathrm{~g} / \mathrm{L} & 46,4 \\ \text { Ellagitan Liquid } & 0,25 \mathrm{~g} / \mathrm{L} & 40,6 \\ & 0,50 \mathrm{~g} / \mathrm{L} & 43,5 \\ \text { Ellagitan Barrique } & 0,25 \mathrm{~g} / \mathrm{L} & 43,8 \\ \text { Ellagitan Pó } & 0,50 \mathrm{~g} / \mathrm{L} & 52,2 \\ & 0,25 \mathrm{~g} / \mathrm{L} & 43,4 \\ & 0,50 \mathrm{~g} / \mathrm{L} & 49,3\end{array}$

Os dados apresentados na tabela 2 permitem inferir que:

- o aumento da acidez se dá de forma diferenciada para cada um dos produtos testados, sendo que os tratamentos com Ellagitan Barrique mostraram maior valor de acidez ao final do período e os tratamentos com Ellagitan Chene mostraram menor ganho de acidez em relação à cachaça branca;

- quanto à relação entre dose de extrato e acidez da cachaça, como esperado, o uso de doses maiores de extratos elevou a acidez na aguardente;

- há uma relação entre cor e acidez, sendo que os tratamentos com maior acidez são os que apresentaram maiores ganhos de cor (FIGURA 2). Essa relação pode ser melhor identificada quando da avaliação de outros atributos por meio da análise sensorial.

\section{Análise sensorial da cachaça adicionada de extratos de carvalho}

O uso de extrato na produção de aguardente composta tem como principal objetivo alterar as características sensoriais do produto, melhorando seus atributos em relação à cachaça branca. Isso pode ser observado nos resultados da análise sensorial apresentados na tabela 3, que mostram diferenças no efeito da adição de cada um dos extratos. 
TABELA 3 - Médias dos resultados obtidos da análise sensorial. Resultados expressos em notas atribuídas pelos julgadores em uma escala de 0 a 10

Table 3 - Average obtained from the sensorial analysis. Notes ( 0 -10 scale) attributed by judges

\begin{tabular}{lllll}
\hline ANÁLISE & Chene & Barrique & Liquid & Pó \\
\hline $\begin{array}{llll}\text { Análise visual } \\
\text { Análise olfativa }\end{array} \quad$ Intensidade & 5,25 & 7,25 & 7,00 & 6,50 \\
$\quad$ Fineza & 6,00 & 7,50 & 6,25 & 6,50 \\
$\quad$ Franqueza & 5,75 & 8,00 & 6,50 & 7,00 \\
Análise gustativa & 6,00 & 7,25 & 6,00 & 6,00 \\
$\quad$ Harmonia & 6,00 & 7,00 & 7,00 & 7,00 \\
$\quad$ Equilíbrio & 4,75 & 6,50 & 6,25 & 6,50 \\
$\quad$ Análise Retro-olfativa & & & & \\
$\quad$ Fineza & 5,50 & 6,25 & 5,75 & 6,00 \\
$\quad$ Fragrância & 5,00 & 6,25 & 5,75 & 6,50 \\
$\quad$ Persistência & 5,00 & 6,00 & 6,75 & 6,75 \\
Impresão Geral & 5,75 & 7,25 & 6,75 & $\mathbf{6 6 , 2 5}$ \\
Soma Geral & $\mathbf{5 5 , 0 0}$ & $\mathbf{6 9 , 2 5}$ & $\mathbf{6 4 , 0 0}$ &
\end{tabular}

No atributo visual, a análise dos julgadores indicou que a adição do Ellagitan Barrique foi o tratamento que resultou em maior contribuição. O segundo melhor desempenho foi obtido com Ellagitan Liquid, seguido pelos extratos Ellagitan Pó e Ellagitan Chene. Digno de nota o fato do tratamento com Ellagitan Barrique ter alcançado nota significativamente superior à do último colocado. Além disso, é importante destacar que o desempenho do Barrique pode ser atribuído à cor mais intensa da bebida, obtida com esse extrato (FIGURA 2), embora a análise visual tenha considerado também outros atributos. A cor é o atributo mais importante na definição da aparência do produto.

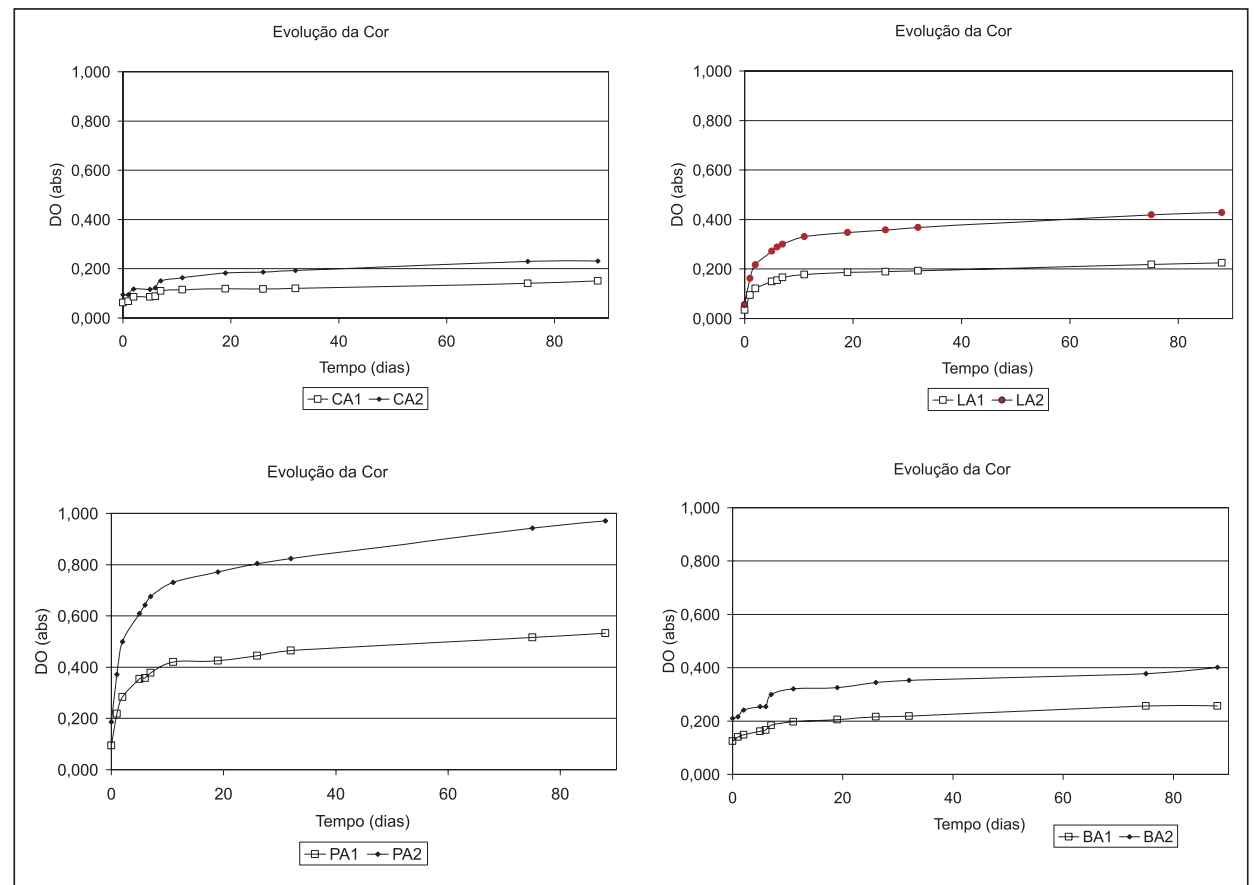

FIGURA 2 - Curvas de incrementos da coloração na aguardente, após adição de extratos de carvalho, em função do tempo

Figure 2 - Curves of coloration increase of composed beverage after oak extracts addition, during time 
Para os atributos referentes à análise olfativa, o Ellagitan Barrique também foi considerado o melhor extrato, alcançando nota média de 7,6 em uma escala de 0-10. Essa nota significa uma melhoria em torno de $15 \%$ em relação ao segundo e aproximadamente $25 \%$ em relação ao último colocado.

Nos dois parâmetros da análise gustativa, harmonia e equilíbrio, houve diferença apenas para um dos produtos testados, sendo que os outros três receberam notas muito próximas. O extrato que obteve as menores notas foi o Ellagitan Chene, com média de 5,4; enquanto que os três outros receberam 6,7. A menor acidez da aguardente elaborada com esse extrato pode ser uma das causas para sua nota baixa.

Conforme a tabela 3, para a análise retro-olfativa, o Ellagitan Pó foi o extrato que mais agradou os julgadores, e, semelhante ao que ocorreu na análise gustativa, o extrato que recebeu as menores notas foi o Ellagitan Chene, sendo que o Liquid e o Barrique obtiveram notas muito próximas.

\section{CONCLUSÕES}

O uso de extratos de carvalho para produção de aguardente composta pode ser considerado uma potencial alternativa para a indústria de bebidas. Apesar de já existirem no mercado aguardentes elaboradas com extratos, esse trabalho mostrou que há uma grande variação nas características das aguardentes em função do extrato e da dose empregadas.

Os quatro extratos de carvalho utilizados neste trabalho promoveram incrementos em coloração na cachaça branca. O produto Ellagitan Chene agregou menos cor à cachaça branca. O extrato Ellagitan Pó contribuiu mais em coloração e foi o extrato com maior estabilidade. Todos os extratos resultaram em aumento de acidez da cachaça. Na análise sensorial, o extrato Ellagitan Barrique foi considerado o extrato que mais contribuiu sensorialmente, seguido do Pó e do Liquid, sendo que o Ellagitan Chene obteve menor desempenho.

\section{AGRADECIMENTOS}

Os autores agradecem a empresa AEB Latino Americana S/A pelos extratos utilizados neste trabalho.

\section{REFERÊNCIAS}

ALMEIDA, J. R.; VALSECHI, O.; NOVAIS, R. F. Envelhecimento das aguardentes: Anais da Escola Superior de Agricultura "Luiz de Queiroz". Piracicaba: v. 4, p.11-83, 1947.

BOWEN, D. et al. Process for preparing an oak wood extract and distillate. USA 5356641, 18 out. 1994. Indopco, Inc. d/b/a Quest International Flovors \& Food Ingredients. Disponível em: patent storn.us/patents/ 5356641. html. Acesso em: 23 set. 2007.

BOZA, Y., OETTERER, M. Envelhecimento de aguardente de cana. Boletim da SBCTA, v. 33, n. 1, p. 8-15, 1999.

BRASIL. Decreto $n^{\circ}$ 4.851, de 2 de outubro de 2003. Altera dispositivos do Regulamento aprovado pelo Decreto $n^{\circ}$ 2.314, de 4 de setembro de 1997, que dispõe sobre a padronização, a classificação, o registro, a inspeção, a produção e a fiscalização de bebidas. Diário Oficial [da] República Federativa do Brasil, Brasília, 3 out. 2003.

CARDELlO, H. M. A. B.; FARIA J. B. Análise descritiva quantitativa da aguardente de cana durante o envelhecimento em tonel de carvalho (Quercus alba l.) Ciênc. Tecnol. Aliment. v. 18, n. 2, maio/jul. 1998. 
CARDELLO, H. M. A. B.; FARIA, J. B. Modificações físico-químicas e sensoriais de aguardente de cana durante o envelhecimento em tonel de carvalho (Quercus alba, L.). Boletim CEPPA, v. 15, n. 2, p. 87-100, 1997.

COUTRIM, M. X.; CHAVES, J. B.; BRANDÃO, R. L. Utilização da microxigenação como alternativa ao processo de envelhecimento da cachaça de alambique. In: EXPOCACHAÇA2003, 2003, Belo Horizonte. Anais ... Belo Horizonte: Rede Mineira de Tecnologia da Cachaça, 2003. p. 24.

DIAS, S.; MAIA, A.; NELSON, D. Efeito de diferentes madeiras sobre a composição da aguardente de cana envelhecida. Ciênc. Tecnol. Aliment. v. 18, p. 169- 175, 1998.

DUARTE, R. M. et al. Analysis of sugar cane spirit submitted to the process of aging in different wood. In: Brazilian Meeting on Chemistry of food and beverages, 3, 2000, São Carlos. Book of Abstracts. São Carlos: Instituto de Química de São Carlos da Universidade de São Paulo, 2000. p. 1.

FERREIRA, V. L. P. Análise sensorial: testes discriminativos e afetivos. São Paulo: SBCTA, p. 109, 1999.

FORLIN, F. J. Maturação de cachaça em recipientes de poliéster com extrato de madeira de carvalho. $174 \mathrm{f}$. Tese (Doutorado em Tecnologia de Alimentos), Faculdade de Engenharia de Alimentos, Universidade Estadual de Campinas, 2005.

MACCARI JR, A. In: Cachaças do Paraná: de gole em gole... da cana ao copo. Curitiba: SEBRAE/PR, p. 104, 2005.

MAIA, A. B. R. A. Tecnologia da cachaça de alambique. Belo Horizonte: SEBRAE/MG; SINDBEBIDAS, 2005. p. 129.

MARTINEZ, R. G. et al. Evolución de los parámetros físico-químicos en aguardientes macerados con madera de roble: influencia del tiempo de maceración. Alimentaria, p.111-117, jul./ago., 1997.

NISHIMURA, K.; MATSUYAMA, R. Maturation and maturation chemistry. In: PIGGOTT, J.R., SHARP, R., DUNCAN, R.E.B. (Ed.). The science and technology of whiskies. New York: Longman Scientific \& Technical, 1989. p. 235-63.

NOVAIS, F. V. Tecnologia das aguardentes. Piracicaba: s/local, 1974.

QUEIROZ-DE, E. L. M. T. Envelhecimento forçado da aguardente de cana: estudo comparativo com o envelhecimento tradicional em carvalho (Quercus sp.). 83 f. Dissertação (Mestrado em Ciência dos Alimentos)-UNESP/Faculdade de Ciências Farmacêuticas, Araraquara, 1998.

REAZIN, G. H. Chemical mechanisms of whiskey maturation. Am. J. Enol. Vitic., v. 32, p. 283-289, 1981. TRITTON, S. M. Spirits, aperitifs and liqueurs: their production. London: Faber and Faber, p. 82, 1975. VALSECHI, O. Envelhecimento da aguardente de cana-de-açúcar. Revista de Tecnologia de Bebidas, v. 4, n. 10, p. 21-29, 1962.

YOKOTA, S. R. C. et al. Mapa de preferência interno de cachaças envelhecidas em barril de carvalho ou tonéis de madeiras brasileiras. In: EXPOCACHAÇA 2003, Belo Horizonte: Rede Mineira de Tecnologia da Cachaça, 2003. p. 27.

ZIMLICH, III; JOSEPH, A. Oak aged alcoholic beverage extract. USA 6132788, 17 out. 2000. Brown Forman Corporation, Louisville, Ky. Disponível em: patent storn.us/patents 6132788. html. Acesso em: 23 set. 2007. 